\title{
Lessons learned from vivo-morpholinos: How to avoid vivo-morpholino toxicity.
}

\author{
David P. Ferguson ${ }^{1,3}$, Lawrence J. Dangott ${ }^{2}$, and J. Timothy Lightfoot ${ }^{3}$ \\ ${ }^{1}$ Children's Nutrition Research Center, Baylor College of Medicine, Houston, TX, ${ }^{2}$ Protein Chemistry \\ Laboratory, Dept. of Biochemistry/Biophysics, Texas A\&M University, College Station, TX, and ${ }^{3}$ Biology of \\ Physical Activity Laboratory, Dept. of Health \& Kinesiology, Texas A\&M University, College Station, TX
}

BioTechniques 56:251-256 (May 2014) doi 10.2144/000114167

Keywords: annexin A6; calsequestrin 1; vivo-morpholino; blood clot formation

Vivo-morpholinos are a promising tool for gene silencing. These oligonucleotide analogs transiently silence genes by blocking either translation or pre-mRNA splicing. Little to no toxicity has been reported for vivo-morpholino treatment. However, in a recent study conducted in our lab, treatment of mice with vivo-morpholinos resulted in high mortality rates. We hypothesized that the deaths were the result of oligonucleotide hybridization, causing an increased cationic charge associated with the dendrimer delivery moiety of the vivo-morpholino. The cationic charge increased blood clot formation in whole blood treated with vivo-morpholinos, suggesting that clotting could have caused cardiac arrest in the deceased mice. Therefore, we investigate the mechanism by which some vivomorpholinos increase mortality rates and propose techniques to alleviate vivo-morpholino toxicity.

The ability to silence genes in vivo provides the opportunity to study gene function in whole animal models and could potentially be used to treat a variety of genetic disorders. Attempts to develop tools for silencing genes in vivo have had limited success. Initially, siRNA and phosphorodiamidate morpholino oligomers (PMOs) were thought to be potential mediators of in vivo gene silencing; however, their use resulted in limited gene silencing and significant associated toxicity (1).

Morcos et al. (2) developed vivomorpholinos as an alternative to siRNA and PMOs. Vivo-morpholinos are antisense oligonucleotide analogs that bind to complementary RNA sequences and block translation of a targeted gene (2). Vivo-morpholinos are unique in that the delivery moiety consists of eight guanidinium head groups (a dendrimer) of arginine-rich peptides, conferring resistance to proteases and nucleases and thus preventing or slowing the degradation of the oligos, increasing the efficiency of their uptake into the cell by endocytosis (2).

A variety of studies have used vivomorpholinos (2-31), and all have reported at least a 50\% knockdown of the target gene with no adverse side effects. Fifteen of these studies used a mouse model $(2,4,11,13,14,16,21,23-30)$, with the remaining studies using rats (17-19), newts (22), chicken embryos (7), zebrafish $(3,5,6)$, and amphibians $(8,10,20)$. In the mouse model, it has been shown that intravenous (IV) and intraperitoneal (IP) administration of vivo-morpholinos were equally efficacious, and recent studies have shown success with direct injection in target tissue (17-19). To this point, no toxicity of vivo-morpholinos has been reported in any published study.

Our lab has evaluated the gene silencing ability and washout effects of vivo-morpholinos in various tissues in a mouse model (29). We have published the effects of three different vivo-morpholinos in addition to a vivo-morpholino cocktail (a combination of two vivo-morpholinos) and achieved significant knockdown of the targeted proteins (Drd1, Glut4, Vmat2) with no observable adverse side effects, even when using the vivo-morpholino cocktail (29). Furthermore, we previously established that the use of a scrambled vivo-morpholino (i.e., a vivomorpholino that does not correspond to any genetic sequence) does not evoke responses different from saline injection (29). Thus, our previous work, as well as the entire vivo-morpholino literature base at this time, has shown vivo-morpholinos to be effective and non-toxic.

Recently, we initiated a study with the goal of using vivo-morpholinos to silence the annexin A6 (Anxa6) and calsequestrin 1 (Casq1) genes via IV injection in the mouse model. Both genes are involved in skeletal muscle calcium regulation. Following our previous success in using vivo-morpholino cocktails (29), we combined the Anxa6 and Casq1 vivo-morpholinos in an attempt to

\section{METHOD SUMMARY}

The purpose of this study was to determine the mechanism by which vivo-morpholino treatment resulted in the death of treated mice. The nucleotide sequences of vivo-morpholinos used in our lab were evaluated for base pairing, and the hybridization of vivo-morpholinos was evaluated in whole blood to determine the influence on blood clot formation. Based on the results, we propose several techniques for preventing vivo-morpholino toxicity. 
silence both genes at the same time. The initial results were disconcerting in that the treatments resulted in numerous fatalities. Thus, the purpose of this report is to characterize the cause of death, mechanism of action, and propose possible solutions for the toxicity we observed with vivo-morpholinos in this model.

\section{Materials and methods}

\section{Observation of the problem}

Vivo-morpholinos (2000 nmol batches) designed to target Anxa6 and Casq1 were obtained from GeneTools LLC (Philomath, OR). GeneTools provides a vivo-morpholino design service whereby the investigator provides the gene of interest along with the $\mathrm{NCBI}$ accession number. Previous work from our lab has utilized the $400 \mathrm{nmol}$ batch of vivo-morpholinos due to the smaller sample sizes of our studies (29). Male C57L/J mice (9 weeks of age) received a tail vein injection of a single vivo-morpholino (11 mg/kg or 56 $\mu \mathrm{L}$ of Anxa6 or Casq1 vivo-morpholino) or a cocktail treatment $(11 \mathrm{mg} / \mathrm{kg}$ of both Anxa6 and Casq1 vivo-morpholino or $\sim 112 \mu \mathrm{L}$ ). These dosages have been recommended by GeneTools (2) and have been used in published studies (31), including our previous work (29), without reports of toxicity. All mice used in this study were C57L/J male mice provided by the Jackson Laboratory (Bar Harbor, ME). Based on the homozygous genetic background of the mice and the fact they were kept in a climate controlled vivarium, the only difference between mice that could explain the differing mortality rates would be the treatment and type of vivomorpholino given.
Following the injections, the mice appeared to recover and were ambulatory within the cage for 1-2 min, followed by an immediate loss of consciousness, an increased breathing rate, and fluid leakage from the nose. Once mice displayed these signs of distress, they were placed on heating pads $\left(37^{\circ} \mathrm{C}\right)$ and given supplemental oxygen (via nose cone). Unfortunately, once a mouse lost consciousness, death occurred within five minutes in all cases. By the time a solution to the problem was determined (as detailed below) and after using several potential solutions provided by GeneTools, a total of 14 out of $17(82 \%)$ animals receiving the vivomorpholino cocktail died, while 6 out of 9 (66\%) of the Casq1 vivo-morpholino treated animals died and 2 out of 8 (25\%) animals receiving the Anxa6 vivo-morpholino died. None of the 15 control (saline injection) animals died.

This level of mortality was disconcerting for several reasons, including financial, logistical, scientific, and most importantly, because of the trauma placed on the animals. While all measures were taken to insure humane treatment of the animals (all studies had been approved by our Institutional Animal Care and Use Committee), the vivo-morpholino treatment resulted in unnecessary stress placed on the mice prior to death. As noted earlier, there have been no reports of similar fatalities as observed in this study. Thus, for further use as a laboratory tool given that potential future applications for vivo-morpholinos may include both non-human primates and human clinical trials, it is critical that the cause of the vivo-morpholino toxicity in this study be understood in order to prevent future fatalities associated with vivo-morpholino treatment.

Potential solutions to the problem Following the fatalities associated with their vivo-morpholinos, GeneTools was consulted. GeneTools hypothesized that the fatalities were a result of guanidinium toxicity and recommended treatment with lower dosages, even though we had previously used identical, recommended dosages without incident (29). Therefore, we subsequently injected C57L/J mice with Casq1, Anxa6, or cocktail vivo-morpholino treatments at a dosage of $4 \mathrm{mg} / \mathrm{kg}$ (reduced from the normal dose of $11 \mathrm{mg} / \mathrm{kg}$ ). This treatment resulted in 8 out of 8 (100\%) fatalities in the cocktail group, along with 3 out of 7 (43\%) fatalities in the Casq1 group, and 1 out of 6 (17\%) fatalities in the Anxa6 group. Thus, in spite of markedly reducing the dose and reducing the exposure to guanidinium in each mouse, we still observed significant mortality rates, which did not support the hypothesis that guanidinium toxicity was the causal factor. Supporting this conclusion is much older literature reporting that the fatal dose of guanidinium is actually 30 times more than the amount the mice received with the vivo-morpholino treatments (32). As we have noted earlier, neither we nor others have observed fatalities with a vivo-morpholino dosage of 11 $\mathrm{mg} / \mathrm{kg}(24,29,31)$.

GeneTools also recommended incubating vivo-morpholinos at high temperature before use. We incubated samples at $121^{\circ} \mathrm{C}$, followed by vortexing prior to use. We then injected the treatment
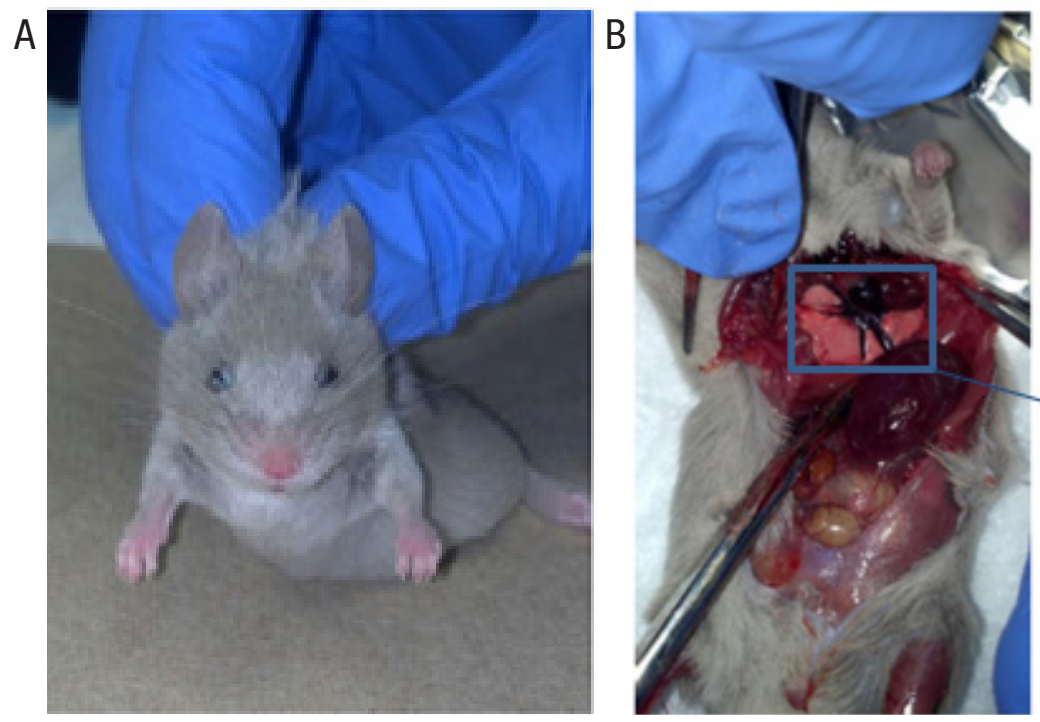

Figure 1. Effects of vivo-morpholino treatment in mice. (A) Photo of vivo-morpholino treatment animal immediately after death. Note the cloudy opaque appearance of the outer edges of the eye. (B) Treatment mouse during necropsy ( 1.5 min post death). The left ventricle was found to be potentially necrotic. (C) Enlarged image of the potentially necrotic left ventricle from panel B.

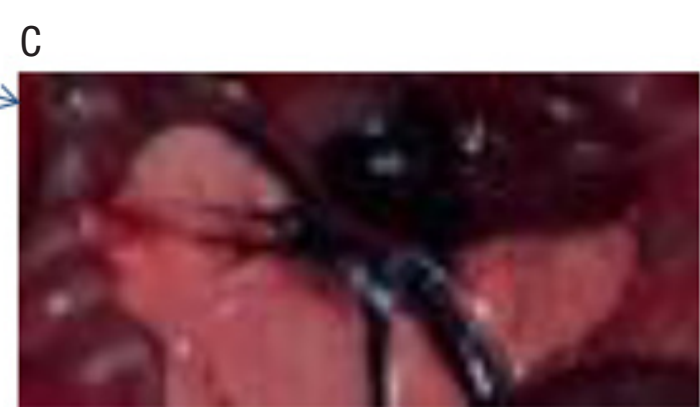


at a temperature of $37^{\circ} \mathrm{C}$. However, this approach did not reduce mortality rates.

Investigation into the cause of death In order to determine the cause of death, mice that died were immediately necropsied with brain, heart, lung, liver, spleen, large intestine, soleus, gastrocnemius, extensor digitorum longus, and kidneys removed. Two initial observations were made during necropsy that set these animals apart from normal anatomical findings during routine dissections: (i) the deceased animals' eyes had a cloudy opaque appearance and (ii) there appeared to be necrotic tissue in the left ventricle of the heart with an increased blood thickness in the vena cava and aorta (Figure 1). At the time, our lab was not equipped to evaluate characteristics of the heart by histological methods. Therefore, we can only speculate that the cause of death from vivo-morpholino treatment was due to cardiac arrest. These observations were common across all animals that died after vivo-morpholino treatment. Similar effects have not been previously documented nor have we observed these symptoms in our previous vivo-morpholino studies. Interestingly, one of our previous studies used the Casq1 vivo-morpholino from a $400 \mathrm{nmol}$ batch, and we did not observe an increased mortality rate in those mice. Thus, we initially hypothesized that the mechanism of action for mortality was due to an unknown, unique factor related to the synthesis of the $2000 \mathrm{nmol}$ product.

The mice used in the $400 \mathrm{nmol}$ and 2000 nmol studies were 9 week old inbred C57L/J male mice. There was no difference in weight or percent body fat in the mice between these two studies (data not shown). Given that the C57L/J mice we used are inbred generation 250 (http://jaxmice.jax.org/strain/000668. $\mathrm{html})$, we are confident that they are genetically homozygous. Furthermore, one reason we picked this strain is their relatively small within strain variation in daily physical activity (33-35). Thus, we conclude that there is no physiological, biological, or environmental difference between these mice that would account for the increased mortality rate other than the different treatments the mice received. The mice received a dosage of the same concentration of Casq1 vivomorpholino; however, animals treated with the $400 \mathrm{nmol}$ batch had physiological saline added to the vivo-morpholino. The 2000 nmol batch was not mixed with physiological saline.

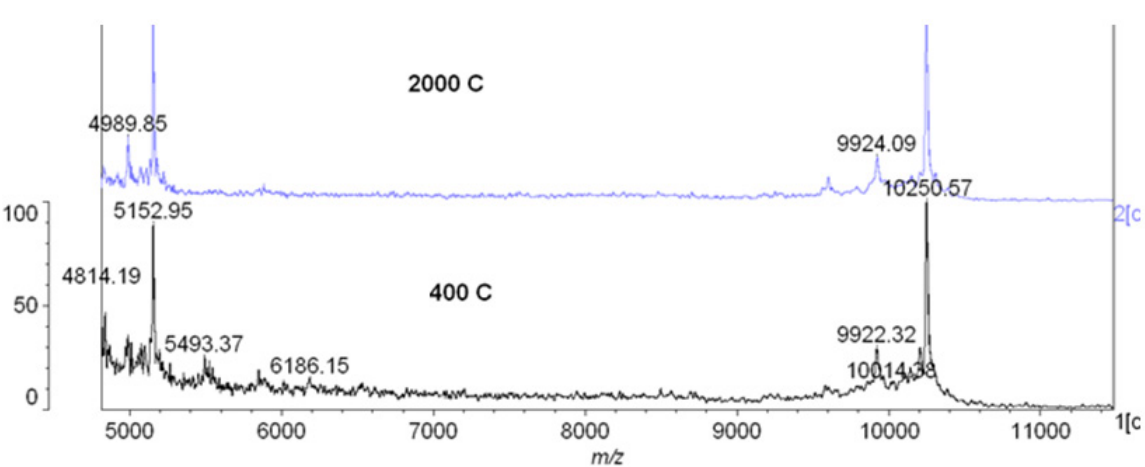

Figure 2. MALDI-TOF results of Casq1 vivo-morpholinos from separate $400 \mathrm{nmol}$ and $2000 \mathrm{nmol}$ batches. There was no difference between the spectra.

To further investigate potential differences in the composition of the vivo-morpholinos, using standard techniques (36), we evaluated the Casq1 vivo-morpholino from the 2000 (fatality-causing) and 400 (non-fatality) nmol concentrations using Matrix Assisted laser desorption/ionization time of flight (MALDITOF) mass spectrometry, with sinapinic acid as the matrix, in an effort to identify any potential contaminants in the $2000 \mathrm{nmol}$ vivomorpholino. While there were peaks in the lower molecular weight range, we dismissed these peaks as contaminants because they were similar to the quality control report generated by GeneTools. While we could not identify these peaks, we suspect they were salt adducts, given that salt adducts are usually $\mathrm{Na}^{+}$or $\mathrm{K}^{+}$. They are naturally occurring and result from salts in the sample (either not removed during purification or added when dissolved in PBS) and are probably not toxic. Indeed, they may only have been detected in the mass spectrum because the sample was crystallized. The adducts may not exist when the vivo-morpholino is in solution, as the salts often dissociate. Therefore, it is unlikely they are toxic. Thus, we observed no difference in the spectra between the 2000 or 400 nmol Casq1 vivo-morpholinos (Figure 2). Furthermore, the spectra were not different from the spectra provided by GeneTools in the quality control report.

Following the MALDI-TOF results, we revisited the necropsy results, especially the suspected necrosis of the left ventricle and increased blood thickness in the vena cava and aorta. Given these observations, we hypothesized that vivo-morpholinos caused significant blood clotting in the heart and vessels, resulting in markedly decreased oxygen delivery to the heart and possibly myocardial infarction.

To test this hypothesis, an experiment evaluating the effects of vivo-morpholinos on blood clotting was conducted. $100 \mu \mathrm{L}$ blood aliquots were mixed with amounts proportional to those used in the study of saline (2.6 $\mu \mathrm{L}, \mathrm{n}=3$ ), vivo-morpholino scramble (2.6 $\mu \mathrm{L}, \mathrm{n}=3$ ), Anxa6 vivo-morpholino (2.6 $\mu \mathrm{L}, \mathrm{n}=3)$, Casq1 vivo-morpholino $(2.6 \mu \mathrm{L}$, $\mathrm{n}=3)$, Anxa6/Casq1 cocktail (5.2 $\mu \mathrm{L}, \mathrm{n}=$ 3), and Drd1/Glut4 cocktail (5.2 $\mu \mathrm{L}, \mathrm{n}=3)$. We used the vivo-morpholino scramble to determine if the delivery vehicle alone influenced blood clot formation. Additionally, we included a Drd1/G/u4 cocktail trial because previous experiments from our lab using this cocktail treatment did not result in any deaths (29). The blood aliquots mixed with the varying treatments were subjected to blood coagulation analysis using a modified version of the light transmission method as outlined by Lim et al. (37). Briefly the blood samples had transmitted light passed through the sample. The transmitted light was detected by a photodiode that was linked to a data acquisition system. As red blood cell aggregation increased, transmitted light decreased, with the threshold time at which transmitted light decreases correlated to blood clot formation time. Statistical analysis was performed in JMP v.7.0 (Cary, NC) using ANOVA to compare multiple means. An alpha level of 0.05 was set a priori. The results showed that blood clotting time was significantly less $(P=0.01)$ in the Casq1, Anax6, and Casq1/Anax6 cocktail vivo-morpholinos (those vivo-morpholinos with significant sequence complementarity) (Figure 3) as compared with the saline, scramble, and Drd1/Glut4 cocktails (those treatments without sequence complementarity) (Figure 4). These results indicate that the vivo-morpholinos with significant ( $>4$ bases) sequence complementarity caused significantly accelerated clotting, suggesting that treatment with these vivo-morpholinos resulted in blood clot formation and possible cardiac death. 


\section{TAKE ACTION AGAINST CONTAMINATION WITH $\mathrm{QMI}^{\circledR}$ SAFE-SEPTUM}

Sample, Inoculate Or Add Nutrients To Your Bioreactor Aseptically!

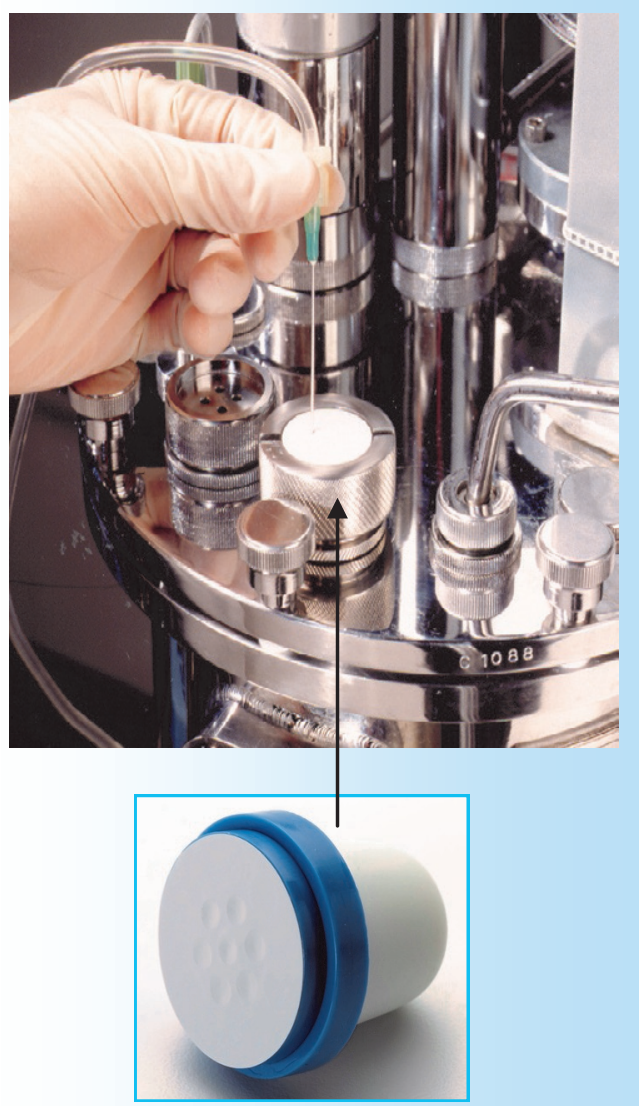

The QMI Safe-Septum is:

- Aseptic

- Pressure \& Temperature Safe

- Pre-Sterilized

- Easy To Retrofit

- Validated

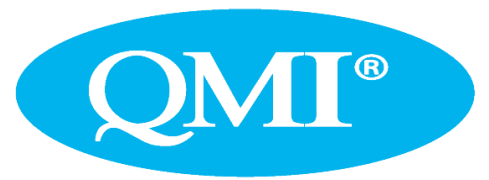

$651-501-2337$

Email: info@qmisystems.com

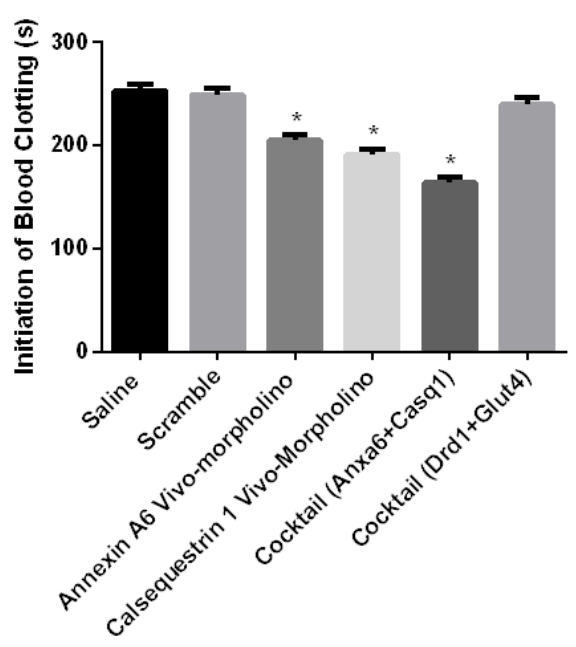

Figure 3. Clotting time of whole blood treated with vivo-mopholinos. Treatments tested included saline, vivo-morpholino scramble, Casq1 vivomorpholino, Anxa6 vivo-morpholino, Anxa6/ Casq 1 vivo-morpholino cocktail, and Drd1/ Glut4 vivo-morpholino cocktail. There was decreased $(P=0.01)$ clotting time for Casq 1 vivomorpholino, Anxa6 vivo-morpholino, and Anxa6/ Casq1 vivo-morpholino cocktail as compared with the clotting time of the other treatments.

\section{Results and discussion}

With our results showing a reduced clotting time with vivo-morpholino treatment and the increased blood viscosity observed in the necropsied vivo-morpholino-treated animals, we hypothesized that the structure of the vivo-morpholino was causing a significant increase in blood clot formation. Due to the fact that a larger number of the fatalities occurred in the cocktail treatment group, we examined the sequences of the Casq1 and Anxa6 vivo-morpholinos. As noted earlier, the sequences chosen for the oligo-portion of the vivo-morpholino are generally chosen by GeneTools (as was the case in our previous studies). When examining the sequences of the supplied Casq1 and Anxa6 vivo-morpholinos, we noted that there were nine nucleotide bases that could hybridize when the sequences were arranged by aligning the 3 ' and 5' ends (Figure 4). Furthermore, there were four potential self-hybridizing pairs in the Casq1 vivo-morpholino and three in the Anxa6 vivo-morpholino (Figure 4). In checking the sequences of the vivo-morpholinos used in our non-fatal experiments (29), we found that the oligos employed in those studies had no potential for either internal or external 5' to 3' base pairing/hybridization.

While the evidence for $5^{\prime}$ and 3 ' pairing is suggestive of a potential cause of death, we explored other methods of complex formation determination. Using SciTools OligoAnalyzer 3.1, we evaluated complex formation of Anxa6, Casq1, Anxa6/Casq1 cocktail and Drd1/Glut4 cocktail. The results showed that the negative delta $G$ for self-dimer formation for Anxa6 was -56.18 and the value for Casq1 was -41.23. In terms of hetero-dimer formation, the Anxa6/Casq1 cocktail had a delta $G$ of -5.02 and the Drd1/Glut cocktail delta G was -1.56 . These results indicate that the probability of vivo-morpholino complex formation is higher in Anax6 and Casq1 followed by the Anxa6/Casq1 cocktail and lastly Drd1/Glut4. Supporting these data, we did not observe a significant difference in clot formation time between Anxa6, Casq1 and the Anxa6/Casq1 cocktail (Figure 3). However, the Drd1/Glut4 cocktail clotting time was significantly longer than the other vivo-morpholino groups and thus does partially mirror the data obtained from SciTools OligoAnalyzer 3.1. It is important to note that SciTools OligoAnalyzer 3.1 does not take into account the morpholino ring structure, which could potentially account for discrepancies between the number of animal deaths and negative delta $G$ values. Thus, we suggest the that vivomorpholinos hybridized forming a complex consisting not only of the hybridized oligos but also the highly charged delivery moiety (dendrimer) and that the high charge density of the dendrimer cluster was responsible for the observed increased blood viscosity that led to the possible ischemic state of the heart and resulting fatality (38). This suggestion is consistent with the observation that those vivo-morpholinos with the highest number of potential hybridization sites (cocktail with 9 hybridization sites) had the highest percentage of fatalities (14 out of 17), vivo-morpholinos with a lower number of hybridization sites (Anxa6 with 3 sites) had a lower number of fatalities (2 out of 8), and those vivo-morpholinos (Drd1) Glut4) that had no potential hybridization sites had no fatalities.

This hypothesized mechanism by which the dendrimer cluster induced fatality is supported by the work of Roberts et al. (39), who investigated the toxic effects associated with dendrimer administration in vivo, specifically the effects of dendrimer generation on in vivo function. The study showed that the increased number of branches associated with the dendrimer (over 10,000 Daltons) increased the sedimentation rates of red blood cells 
(39). The increased number of cationic dendrimer head groups associated with the increased branch number of the dendrimer altered the surface charge density of the membranes of the red blood cells, causing a conformational change in the membrane $(39,40)$ resulting in increased blood viscosity and blood clotting, which led to possible myocardial infarction (38). Thus, hybridization of vivo-morpholinos with multiple potential hybridization sites would lead to a significantly increased dendrimer clustering, which would lead to a reduced clotting time and possible cardiac death.

The easiest solution to the toxicity issue is to take care when designing the vivo-morpholinos to insure little to no hybridization potential. GeneTools allows customers to design their own oligos, and we recommend this option as opposed to the algorithm generated vivo-morpholinos provided by GeneTools, especially if the experiment uses multiple vivo-morpholinos given in cocktail injections. Furthermore, use of SciTools OligoAnalyzer 3.1 to test for potential hybridization will be valuable to vivo-morpholino design. However,

\section{A: Cocktail Treatment}

\section{Anxa6}

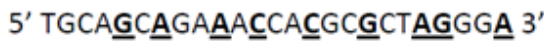

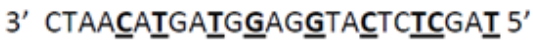

Casq1

\section{B: Calsequestrin 1 Treatment}

\section{5' TㅁGCTCTㅁATGGAGGTAG TACAAIC 3'}

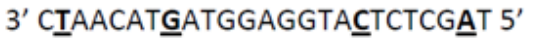

\section{C: Annexin A6 Treatment}

\section{5' IGCAGCAGAAACCACGCGCTAG $\underline{G} \underline{\text { A 3' }}$}

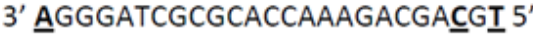

Figure 4. Nucleotide base pairing of $5^{\prime}$ and $3^{\prime}$ ends of the vivo-morpholinos. Bold and underlined nucleotides indicate the location of base pairing. There was a direct relationship between the number of nucleotide base pairs and the number of fatalities. A total of 14 out of 17 (82\%) animals receiving the vivo-morpholino cocktail died, while 6 out of $9(66 \%)$ of the Casq 1 vivo-morpholino treated animals died, and 2 out of $8(25 \%)$ animals receiving the Anxa6 vivo-morpholino died. there are situations, such as ours, where targeted genes (single vivo-morpholino) may have similar sequences and some potential hybridization may occur. While we recommend whenever possible to use careful oligo design, one potential solution to alleviate dendrimer induced red blood cell sedimentation is to add an equal volume of physiological saline to the vivomorpholino injection and vortex vigorously before injection. By employing this simple procedure, we eliminated fatalities in the mice that received these treatments $(n=6)$, yet still observed significant gene silencing. We hypothesize that the addition of physiological saline (specifically the chloride ions) prevents red blood cell aggregation by masking the positive charges on the arginine-rich dendrimer. In fact, we think this is why the $400 \mathrm{nmol}$ Casq1 batch did not initially result in any fatalities. The only difference in the treatments was that the 400 nmol injection was mixed with physiological saline to maintain volume for varying dosage amounts.

Anticoagulation therapy should also be considered as a possible treatment for vivomorpholino induced blood clot formation. Further testing of the appropriate dosage and treatment method is required to fully understand the potential benefits of anticoagulation therapy. However, we feel that appropriate vivo-morpholino design and addition of physiological saline could potentially eliminate the need for anticoagulation.

In conclusion, while vivo-morpholinos are an ideal tool for transient gene silencing, caution should be taken in oligo design to avoid dendrimer induced red blood cell sedimentation. In order to prevent fatalities, it is recommended that the oligonucleotide analogs be analyzed for potential $3^{\prime}$ to $5^{\prime}$ base pair hybridization in order to prevent clustering of dendrimers. If that is not possible, then addition of physiological saline to the vivo-morpholino or use of anticoagulation therapy may alleviate toxicity.

\section{Author Contributions}

D.F. wrote the manuscript, observed the blood clotting, tested clotting time, and experimented with addition of physiological saline. L.D. ran the MALDI-TOF experiment, consulted on the mechanism by which blood clots could form, and reviewed the manuscript. T.L. funded the project and reviewed and edited the manuscript

\section{Acknowledgments}

The authors thank Paul Morcos and Joe Morton of GeneTools LLC for the extensive conversations regarding the issues addressed in this article. Additionally the authors thank Brooks Scull and Ryan Fleischmann of Baylor College of Medicine for assisting in the blood clotting experiments. This project was funded by $\mathrm{NIH}$ AR050085 and start-up funds from the Department of Health and Kinesiology at Texas A\&M University. This paper is subject to the $\mathrm{NIH}$ Public Access Policy.

\section{Competing interests}

The authors declare no competing interests.

\section{References}

1. Stein, D.A. 2008. Inhibition of RNA virus infections with peptide-conjugated morpholino oligomers. Curr. Pharm. Des. 14:2619-2634.

2. Morcos, P.A., Y. Li, and S. Jiang. 2008. VivoMorpholinos: a non-peptide transporter delivers Morpholinos into a wide array of mouse tissues. Biotechniques 45:613-618.

3. Guo, Y., L. Ma, M. Cristofanilli, R.P. Hart, A. Hao, and M. Schachner. 2011. Transcription factor Sox $11 \mathrm{~b}$ is involved in spinal cord regeneration in adult zebrafish. Neuroscience 172:329-341.

4. Kang, J.K., A. Malerba, L. Popplewell, K. Foster, and G. Dickson. 2011. Antisense-induced myostatin exon skipping leads to muscle hypertrophy in mice following octa-guanidine morpholino oligomer treatment. Mol. Ther. 19:159-164.

5. Kim, S., U.P. Radhakrishnan, S.K. Rajpurohit, V. Kulkarni, and P. Jagadeeswaran. 2010. Vivo-Morpholino knockdown of alphallb: A novel approach to inhibit thrombocyte function in adult zebrafish. Blood Cells Mol. Dis. 44:169-174.

6. Kizil, C. and M. Brand. 2011. Cerebroventricular microinjection (CVMI) into adult zebrafish brain is an efficient misexpression method for forebrain ventricular cells. PLoS ONE 6:e27395.

7. Kowalik, L. and A.J. Hudspeth. 2011. A search for factors specifying tonotopy implicates DNER in hair-cell development in the chick's cochlea. Dev. Biol. 354:221-231.

8. Liu, Y., H. Yu, S.K. Deaton, and B.G. Szaro. 2012. Heterogeneous nuclear ribonucleoprotein $\mathrm{K}$, an RNA-binding protein, is required for optic axon regeneration in Xenopus laevis. J. Neurosci. 32:3563-3574.

9. Maki, N., R. Suetsugu-Maki, S. Sano, K. Nakamura, O. Nishimura, H. Tarui, K. Del Rio-Tsonis, K. Ohsumi, et al. 2010. Oocyte-type linker histone B4 is required for transdifferentiation of somatic cells in vivo. FASEB J. 24:3462-3467.

10. Matsuda, H. and Y.B. Shi. 2010. An essential and evolutionarily conserved role of protein arginine methyltransferase 1 for adult intestinal stem cells during postembryonic development. Stem Cells 28:2073-2083.

11. Nazmi, A., K. Dutta, and A. Basu. 2010. Antiviral and neuroprotective role of octaguanidinium dendrimer-conjugated morpholino oligomers in Japanese encephalitis. PLoS Negl. Trop. Dis. 4:e892. 


\section{Find the PEANUT}

12. Nazmi, A., R. Mukhopadhyay, K. Dutta, and A. Basu. 2012. STING Mediates Neuronal Innate Immune Response Following Japanese Encephalitis Virus Infection. Sci Rep. 2:347.

13. Osorio, F.G., C.L. Navarro, J. Cadinanos, I.C. Lopez-Mejia, P.M. Quiros, C. Bartoli, J. Rivera, J. Tazi, et al. 2011. Splicing-directed therapy in a new mouse model of human accelerated aging, Sci Transl Med. 106:106ra107.

14. Owen, L.A., H. Uehara, J. Cahoon, W. Huang, J. Simonis, and B.K. Ambati. 2012. Morpholinomediated increase in soluble Flt-1 expression results in decreased ocular and tumor neovascularization. PLoS ONE 7:e33576.

15. Parra, M.K., T.L. Gallagher, S.L. Amacher, N. Mohandas, and J.G. Conboy. 2012. Deep intron elements mediate nested splicing events at consecutive AG dinucleotides to regulate alternative 3's splice site choice in vertebrate 4.1 genes. Mol. Cell. Biol. 32:2044-2053.

16. Parra, M.K., S. Gee, N. Mohandas, and J.G. Conboy. 2011. Efficient in vivo manipulation of alternative pre-mRNA splicing events using antisense morpholinos in mice. J. Biol. Chem. 286:6033-6039.

17. Quinn, M., Y. Ueno, H.Y. Pae, L. Huang, G. Frampton, C. Galindo, H. Francis, D. Horvat, et al. 2012. Suppression of the HPA axis during extrahepatic biliary obstruction induces cholangiocyte proliferation in the rat. Am. J. Physiol. Gastrointest. Liver Physiol. 302:G182-G193.

18. Reissner, K.J., G.C. Sartor, E.M. Vazey, T.E. Dunn, G. Aston-Jones, and P.W. Kalivas. 2012. Use of vivo-morpholinos for control of protein expression in the adult rat brain. J. Neurosci. Methods 203:354-360.

19. Sartor, G.C. and G.S. Aston-Jones. 2012. A septalhypothalamic pathway drives orexin neurons, which is necessary for conditioned cocaine preference. J. Neurosci. 32:4623-4631.

20. Shi, Y.B., T. Hasebe, L. Fu, K. Fujimoto, and A. Ishizuya-Oka. 2011. The development of the adult intestinal stem cells: Insights from studies on thyroid hormone-dependent amphibian metamorphosis. Cell Biosci. 1:30.

21. Taniguchi-Ikeda, M., K. Kobayashi, M. Kanagawa, C.C. Yu, K. Mori, T. Oda, A. Kuga, H. Kurahashi, et al. 2011. Pathogenic exon-trapping by SVA retrotransposon and rescue in Fukuyama muscular dystrophy. Nature 478:127-131.

22. Tsonis, P.A., T. Haynes, N. Maki, K. Nakamura, M.M. Casco-Robles, S. Yamada, T. Miura, C. Chiba, and K. Del Rio-Tsonis. 2011. Controlling gene loss of function in newts with emphasis on lens regeneration. Nat. Protoc. 6:593-599.

23. Vera, T. and D.E. Stec. 2010. Moderate hyperbilirubinemia improves renal hemodynamics in ANG Il-dependent hypertension. Am. J. Physiol. Regul. Integr. Comp. Physiol. 299:R1044-R1049.

24. Widrick, J.J., S. Jiang, S.J. Choi, S.T. Knuth, and P.A. Morcos. 2011. An octaguanidine-morpholino oligo conjugate improves muscle function of $\mathrm{mdx}$ mice. Muscle Nerve 44:563-570.

25. Wu, B., E. Benrashid, P. Lu, C. Cloer, A. Zillmer, M. Shaban, and Q.L. Lu. 2011. Targeted skipping of human dystrophin exons in transgenic mouse model systemically for antisense drug development. PLoS ONE 6:e19906.

26. Wu, B., Y. Li, P.A. Morcos, T.J. Doran, P. Lu, and Q.L. Lu. 2009. Octa-guanidine morpholino restores dystrophin expression in cardiac and skeletal muscles and ameliorates pathology in dystrophic mdx mice. Mol. Ther. 17:864-871

27. Zammarchi, F., E. de Stanchina, E. Bournazou, T. Supakorndej, K. Martires, E. Riedel, A.D. Corben, J.F. Bromberg, and L. Cartegni. 2011.
Antitumorigenic potential of STAT3 alternative splicing modulation. Proc. Natl. Acad. Sci. USA 108:1777917784.

28. Azoitei, N., G.V. Pusapati, A. Kleger, P. Moller, R. Kufer, F. Genze, M. Wagner, J. van Lint, et al. 2010. Protein kinase D2 is a crucial regulator of tumour cell-endothelial cell communication in gastrointestinal tumours. Gut 59:1316-1330.

29. Ferguson, D.P., E.E. Schmitt, and J.T. Lightfoot. 2013. Vivo-Morpholinos Induced Transient Knockdown of Physical Activity Related Proteins. PLoS ONE 8:e61472.

30. Wu, B., P. Lu, E. Benrashid, S. Malik, J. Ashar, T.J. Doran, and Q.L. Lu. 2010. Dose-dependent restoration of dystrophin expression in cardiac muscle of dystrophic mice by systemically delivered morpholino. Gene Ther. 17:132-140.

31. Notch, E.G., J.R. Shaw, B.A. Coutermarsh, M. Dzioba, and B.A. Stanton. 2011. Morpholino gene knockdown in adult Fundulus heteroclitus: role of SGK1 in seawater acclimation. PLoS ONE 6:e29462.

32. National Institute for Occupational Safety and Health., Tracor Jitco Inc., Advanced Engineering \& Planning Corp. Registry of toxic effects of chemical substances, 1977-19: DHEW publication no (NIOSH), U.S. Dept. of Health, Education, and Welfare, Public Health Service, Center for Disease Control Supt. of Docs., U.S. G.P.O., Cincinnati, Ohio Washington, D.C., pp. microfiches.

33. Lightfoot, J.T., L. Leamy, D. Pomp, M.J. Turner, A.A. Fodor, A. Knab, R.S. Bowen, D. Ferguson, et al. 2010. Strain screen and haplotype association mapping of wheel running in inbred mouse strains. J. Appl. Physiol. 109:623-634

34. Lightfoot, J.T., M.J. Turner, M. Daves, A. Vordermark, and S.R. Kleeberger. 2004. Genetic influence on daily wheel running activity level. Physiol. Genomics 19:270-276.

35. Lightfoot, J.T., M.J. Turner, D. Pomp, S.R. Kleeberger, and L.J. Leamy. 2008. Quantitative trait loci for physical activity traits in mice. Physiol. Genomics 32:401-408.

36. Fagerquist, C.K., B.R. Garbus, K.E. Williams, A.H. Bates, and L.A. Harden. 2010. Covalent attachment and dissociative loss of sinapinic acid to/from cysteinecontaining proteins from bacterial cell lysates analyzed by MALDI-TOF-TOF mass spectrometry. J. Am. Soc. Mass Spectrom. 21:819-832.

37. Lim, H., J. Nam, Y. Lee, S. Xue, S. Chung, and S. Shin. Coagulation Study Using Light-Transmission Method, 14th Intrenational Conference on Miniaturized Systems for Chemistry and Life Sciences (2010) 803-806.

38. Gordon, R.J., G.K. Snyder, H. Tritel, and W.J. Taylor. 1974. Potential significance of plasma viscosity and hematocrit variations in myocardial ischemia. Am. Heart J. 87:175-182.

39. Roberts, J.C., M.K. Bhalgat, and R.T. Zera. 1996 Preliminary biological evaluation of polyamidoamine (PAMAM) Starburst dendrimers. J. Biomed. Mater. Res. 30:53-65.

40. Klajnert, B. and M. Bryszewska. 2001. Dendrimers: properties and applications. Acta Biochim. Pol. 48:199-208.

Received 05 June 2013; accepted 26 March 2014.

Address correspondence to David P. Ferguson Children's Nutritional Research Center, Baylor College of Medicine, Houston, TX. E-mail: David.ferguson@ bcm.edu

To purchase reprints of this article, contact: biotechniques@fosterprinting.com 